\title{
From incus bypass to malleostapedotomy: technical improvements and results
}

\author{
A RAMBOUSEK, C H SCHLEGEL, T E LINDER \\ Department of Otorhinolaryngology - Head and Neck Surgery, Kantonsspital Luzern, Lucerne, Switzerland
}

\begin{abstract}
Objective: To assess results of malleostapedotomy using a Fisch Storz titanium piston with at least 10 months' follow up.

Methods: Using a prospective database, the indications, surgical technique, and pre- and post-operative audiometric data for 60 patients undergoing malleostapedotomy between 2002 and 2010 were evaluated. Diagnoses and primary and revision surgeries were compared with reference to the literature.

Results: Sixty endaural malleostapedotomies were performed, 28 as a primary intervention and 32 as revision surgery. In 68 per cent, the underlying pathology was otosclerosis. The most common reason for revision surgery (i.e. in 59 per cent) was prosthesis dysfunction. Overall, the mean air-bone gap $(0.5-3 \mathrm{kHz})$ for the primary intervention and revision surgery groups was 9.4 and $11.3 \mathrm{~dB}$, respectively; an air-bone gap of less than $20 \mathrm{~dB}$ was obtained in 100 and 81 per cent of patients, respectively. There was no significant audiological difference between the primary and revision surgeries groups, and no deafness.

Conclusion: Malleostapedotomy shows comparable results to standard incus-stapedotomy and may be preferable in the presented situations.
\end{abstract}

Key words: Conductive Hearing Loss; Malleus; Otosclerosis; Stapes Surgery; Otologic Surgical Procedures; Ossicular Replacement Prosthesis; Treatment Outcome

\section{Introduction}

Due to continuous improvements in surgical techniques, instrumentation and prosthesis design, otologists can now manage many different types of ossicular pathology.

However, with long-term follow up, revision surgery may become necessary because of prosthesis failure, bone resorption, overlooked primary pathology or recurrent middle-ear disease. Revision surgery performed after unsuccessful fenestrations or stapedectomy led to the introduction of the incus replacement or incus bypass procedure using a malleus grip prosthesis in cases of incus or malleus head pathology. In the 1980s, extensive surgical series using stainless steel fat-wire and Teflon-wire prostheses were presented by Schuknecht and Bartley and Sheehy.,2 These authors' technique was surgically challenging: using an ear speculum via a transcanal approach, the wire of the incus replacement prostheses had to be wrapped around the midportion of the malleus handle, after first elevating the tympanic membrane from the lateral surface of the malleus. The results of such incus replacement procedures were favourable but the risks to the inner ear were significant. As a result, the technique (also known as malleovestibulopexy, malleostapediopexy and malleus attachment stapedectomy) was seldom used (Sheehy reported its use in only 2 per cent of primary stapedectomy cases).

However, following the introduction of three major improvements by Fisch, May and colleagues, ${ }^{3,4}$ the technique (termed malleostapedotomy by Fisch) was revived. These improvements were as follows.

Firstly, an endaural incision was used in combination with a large tympanomeatal flap, allowing a partial anterosuperior canalplasty which enabled better exposure of the upper malleus, including the anterior malleal process and ligament. ${ }^{5}$

Secondly, only limited elevation of the tympanic membrane was performed, $1 \mathrm{~mm}$ caudal to the lateral malleal process, and the prosthesis loop was crimped as close as possible to the lateral malleal process. In this way, the substantial potential movement of the

Presented at the Annual Spring Conference of the Swiss Society of Oto-Rhino-Laryngology, Head and Neck Surgery, 17-18 June, 2010, Zurich, Switzerland

Accepted for publication 17 April 2012 
malleus umbo (up to $1 \mathrm{~mm}$ during sneezing or rapid changes in barometric pressure) was reduced to less than $0.5 \mathrm{~mm}$.

Thirdly, the introduction of the $8.5 \times 0.4 \mathrm{~mm}$ Fisch titanium stapes prosthesis permitted bending of the prosthesis shaft to achieve a perpendicular position of the stapes piston on the footplate, when the position of the malleus handle was too anterior. (The previously used platinum-Teflon piston seemed not to withstand the stronger movements of the malleus handle over time, and occasionally 'disconnected'.)

Following the introduction of these three improvements, malleostapedotomy has become a common alternative in stapes surgery, and is now used more frequently by some authors; Fisch recently reported using the technique in almost 50 per cent of cases (U Fisch, personal communication).

In the current study, we reviewed our experience of 60 consecutive primary and revision malleostapedotomy procedures conducted by the senior authors (TL and CS) since 2002, and we discuss the underlying pathology and surgical challenges.

\section{Materials and methods}

\section{Patients and evaluation}

From 2002 onwards, all patients undergoing otological surgery were entered into a statistical database (www. innoforce.com) as a case series with planned data collection.

In 2010, we evaluated data from all consecutive patients who had undergone primary or revision malleostapedotomy. The mean follow-up time for these patients was 2.5 years (minimum follow up, 10 months). Informed consent was obtained and the study was approved by the institutional review board (Kantonale Forschungsethikkommission; approval number 933). The pre-operative audiogram was compared to the first and last available post-operative audiograms. Surgical drawings and reports were analysed regarding the indication for primary surgery or revision, any surgical peculiarities were noted, and data for primary and revision procedures were compared. All patients undergoing revision procedures had a pre-operative computed tomography (CT) scan to identify the site, insertion depth and angulation of the current prosthesis, and also to exclude additional middle or inner ear pathology. A minority of primary malleostapedotomy patients had a pre-operative CT scan.

Data were collected, coded and analysed using the Mann-Whitney $\mathrm{U}$ test. A $p$ value of less than 0.05 was considered statistically significant.

\section{Surgical technique}

Most patients were operated upon under general anaesthesia.

An endaural approach was used. The anterior limb of the skin flap was incised far anteriorly to the malleus handle (10 o'clock in a left ear, 3 o'clock in a right ear) to allow sufficient exposure, ${ }^{6}$ whereas the posterior limb was incised close to the 6 o'clock position. In cases with a narrow bony ear canal, a partial canalplasty was performed using diamond drills to enlarge the view over the malleus neck. The tympanomeatal flap was elevated, preserving and separating the chorda from the undersurface of the tympanic membrane. Shrapnell's membrane was lifted laterally and the entire flap was pushed inferiorly to the lateral process of the malleus, exposing the anterior malleal process and ligament. The malleus handle was uncovered $1-2 \mathrm{~mm}$ caudal to the lateral process. The tympanic membrane attachment to the middle and inferior third of the malleus handle remained intact. The middle ear was inspected and the mobility of the ossicles and the footplate verified. Special attention was paid to the mobility of the malleus by palpating the malleus neck and observing the movements of the anterior malleal ligament. In revision cases, the previous stapes prosthesis was removed and the footplate left untouched until prosthesis placement at a later stage. The incus was removed, avoiding damage to the chorda tympani. In cases with a totally or partially fixed malleus, the chorda was separated from the undersurface of the malleus neck and the neck was sectioned using small $(0.6$ or $0.8 \mathrm{~mm})$ diamond drills or a malleus nipper. The head of the malleus and the anterior malleal process with the ligament were removed to allow free mobility of the malleus handle attached to the tympanic membrane. The stump of the malleus neck was still riding on the chorda, and the tensor tympani tendon was always preserved. In cases with normal mobility, the malleus head was left in place.

The distance between the lateral surface of the malleus handle just distal to the lateral process and the footplate was determined using a measuring rod, and a $0.4 \mathrm{~mm}$ Fisch titanium stapes piston (Karl Storz, Tuttlingen, Germany) was trimmed on a cutting block to the proper length (adding $0.5 \mathrm{~mm}$ to the measured length to enable insertion into the vestibule). The piston was introduced into the middle ear parallel to the malleus and perpendicular to the footplate. The proper length and inclination were verified and an adequate bend was made to bring the shaft perpendicular to the footplate. The diameter and form of the malleus are known to be quite variable. ${ }^{7}$ In the majority of cases, the loop of the piston had to be temporarily enlarged by sliding the clip portion along the shaft of a $2.5 \mathrm{~mm}, 45^{\circ}$ hook. Having finalised the correct length, inclination and loop opening of the prosthesis, the piston was re-introduced into the middle ear. Only at this point was the footplate perforated (or reopened in cases of revision surgery) using laser tools (Erbium:YAG, KTP or diode lasers) and manual perforators. The diameter of the stapedotomy opening was approximated to $0.5-0.6 \mathrm{~mm}$ for the $0.4 \mathrm{~mm}$ piston. The piston was now introduced into the stapedotomy opening, and at the same time the loop was 
placed over the malleus handle just distal to the lateral process. In ideal settings, the surgeon was able to see the footplate and the malleus handle at the same time in order to verify the correct placement of the piston. The loop was tightly fixed onto the malleus using straight and angulated micro-alligator forceps (Figure 1).

After proper fixation, the oval window niche was sealed with two to three small pieces of soft tissue from the endaural incision and a few drops of patient's own blood. The tympanomeatal flap was repositioned and the external ear canal packed with a minimal amount of Gelfoam (Gelita; Proreo Pharma, Liestal, Switzerland) and Ivalone pieces (Novimed, Dietikon, Switzerland). The endaural incision was closed primarily.

(a)

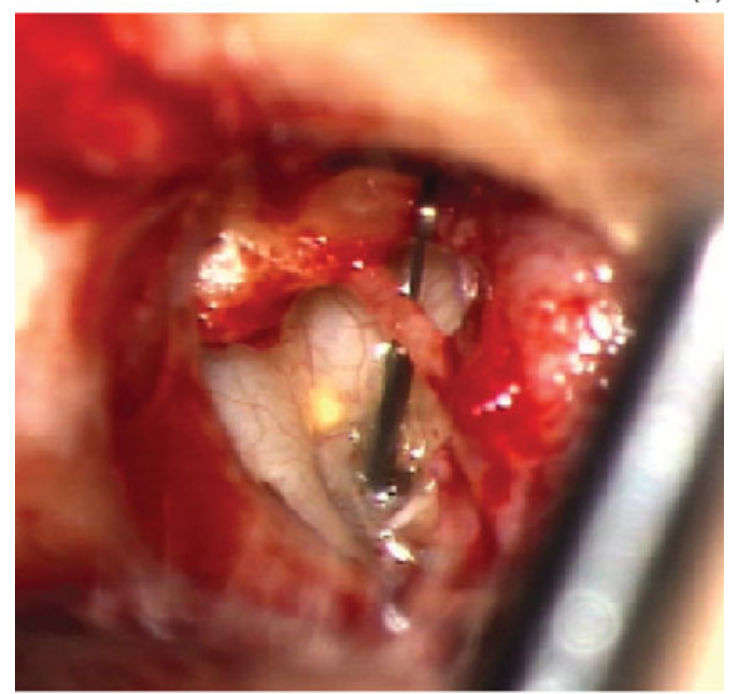

(b)

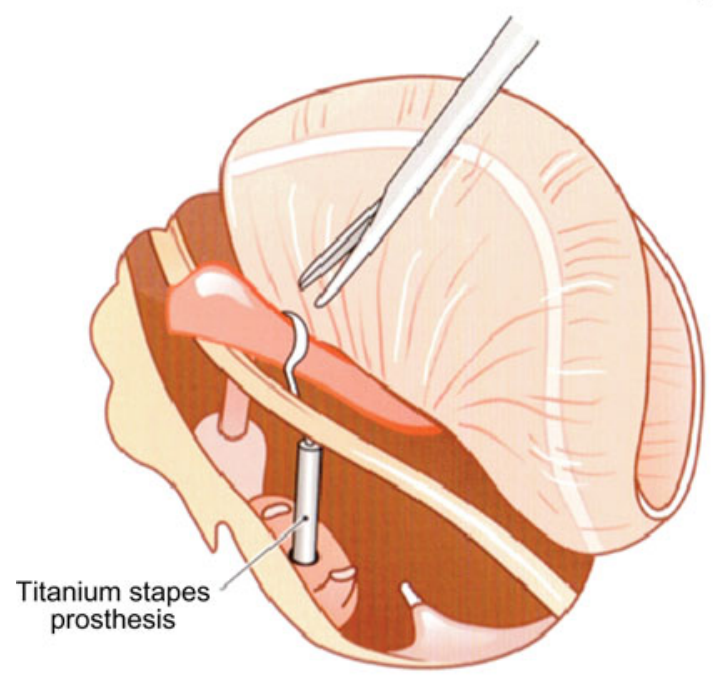

FIG. 1

(a) Intra-operative view of a Fisch titanium stapes piston attached to the upper malleus handle (with slight bend in its shaft). (b) Diagram of malleostapedotomy after malleus head removal, with a $0.4 \mathrm{~mm}$ diameter prosthesis. (Illustration courtesy of Temporal Bone Dissection, the Zürich Guidelines. ${ }^{14}$ Printed with permission of Karl Storz GMBH \& Co KG, Tuttlingen, Germany.)
Further details of the surgical steps are given in Tympanoplasty, Mastoidectomy and Stapes Surgery ${ }^{6}$ and in articles by Kwok et al. ${ }^{7}$ and Fisch et al. ${ }^{4}$

\section{Results and analysis}

A total of 68 surgical procedures were recorded between 2002 and 2010 .

Eight patients were excluded, for the following reasons: five patients were lost to follow up; two cases had an inner ear malformation with an endolymphatic 'gusher' not known prior to surgery; and one patient had severe mixed hearing loss and underwent subsequent cochlear implantation.

Of the remaining 60 ears, 28 underwent primary malleostapedotomy and 32 revision malleostapedotomy. Sixty-two per cent of patients were female and 38 per cent male. Patient follow-up times ranged from 10 months to 6.5 years, with a mean time of 2.5 years. The mean prosthesis length was $7 \mathrm{~mm}$, with a range from 6 to $8.25 \mathrm{~mm}$.

\section{Primary cases}

In the primary malleostapedotomy cases, the main underlying diagnosis was otosclerosis in 71 per cent, with 3 out of 20 patients having a fixed footplate but an invisible focus. Tympanosclerosis was diagnosed in 11 per cent of cases. Congenital cholesteatoma and middle-ear malformation with a mobile stapes footplate were seen in two patients each. One patient had fractured ossicles and a mobile footplate due to trauma (Table I).

The footplate was fixed in all but 2 of the otosclerosis and tympanosclerosis cases undergoing primary surgery (i.e. in 21 of these cases; 75 per cent of all primary cases). In the other cases, we found a mobile footplate (in 7 cases) and missing (3), fixed (3) or fractured (2) stapes suprastructure (Table II). In addition, a partial or total malleus fixation was observed in 7 of the 20 otosclerosis patients and in 2 of the 3 tympanosclerosis cases. Overall, a malleus fixation was seen in 10 of the 28 primary cases ( 36 per cent). A partial or total incus fixation within the epitympanum was

\begin{tabular}{|c|c|c|c|}
\hline & $\begin{array}{r}\text { TABL } \\
\text { DERLYING }\end{array}$ & $\begin{array}{l}\text { I } \\
\text { IAGNOSIS }\end{array}$ & \\
\hline Diagnosis & Procedu & $(\%(n))$ & Total $(\%(n))$ \\
\hline & Primary & Revision & \\
\hline Otosclerosis* & $71(20 / 28)$ & $66(21 / 32)$ & $68(41 / 60)$ \\
\hline Tympanosclerosis & $11(3 / 28)$ & $13(4 / 32)$ & $12(7 / 60)$ \\
\hline Cholesteatoma & & & $7(4 / 60)$ \\
\hline - Congenital & $7(2 / 28)$ & & \\
\hline - Acquired & & $6(2 / 32)$ & \\
\hline Malformation $^{\dagger}$ & $7(2 / 28)$ & 0 & $3(2 / 60)$ \\
\hline Trauma Tumor & $4(1 / 28)$ & $9(3 / 32)$ & $7(4 / 60)$ \\
\hline Tumor & 0 & $6(2 / 32)$ & $3(2 / 60)$ \\
\hline
\end{tabular}

Data represent patient percentages (numbers of patients with diagnosis/patients in group). ${ }^{*}$ Focus not visible in three patients. ${ }^{\dagger}$ Footplate mobile. 


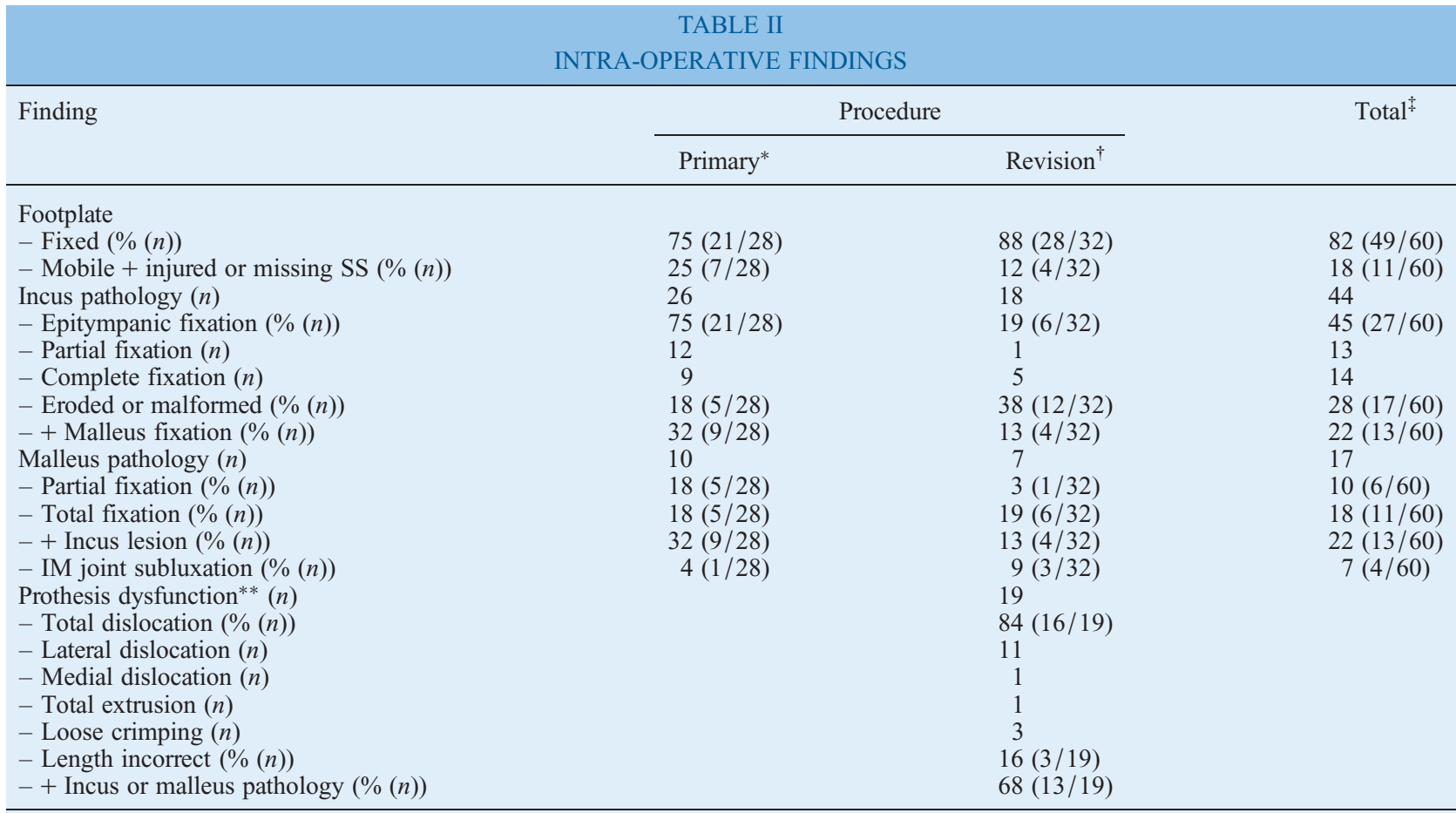

Data represent patient percentages and numbers, as indicated. ${ }^{*} n=28 ;{ }^{\dagger} n=32 ;{ }^{\star} n=60$. ${ }^{* *}$ Twenty-eight of the 32 revision patients had an ossiculoplasty prosthesis placed at previous surgery. $\mathrm{SS}=$ suprastructure; $+=$ combined with; $\mathrm{IM}=$ incudomalleal

identified in 17 of the 20 otosclerosis patients $(85$ per cent) and in all 3 tympanosclerosis cases. This incus fixation was verified by palpating the incus-malleus complex, which identified partial fixation in 12 cases and total fixation in 9 cases. It was further clarified following separation of the incudomalleal joint with a $2.5 \mathrm{~mm}, 45^{\circ}$ angulated hook, leaving the incus remaining tightly fixed within the epitympanum and necessitating forceful luxation of the incus body into the middle ear after shortening the long process to avoid trauma to the chorda tympani nerve. Overall, the incus was fixed in 21 of the 28 primary cases ( 75 per cent). Both incus and malleus fixations were observed in 9 of these 28 cases, including 6 of the 20 otosclerosis patients and 2 of the tympanosclerosis patients.

\section{Revision cases}

In the 32 revision malleostapedotomy cases, the underlying diagnosis was otosclerosis in 66 per cent, tympanosclerosis in 13 per cent, trauma tumor in 9 per cent, and acquired cholesteatoma and tumor in 6 per cent each (Table I).

The most common reason for failure in the 28 procedures conducted on patients with previous ossiculoplasty was prosthesis dysfunction, seen in 19 of these 28 cases (68 per cent; see Table II and below). In 13 of these 19 cases (68 per cent), the previously placed piston had dislocated laterally (11 cases) or medially (1) or had been totally extruded (1); loose crimping was seen in 3 patients. In 3 of the 28 cases (16 per cent), the length was not correct (mainly being too short). In 16 of the 19 cases ( 84 per cent), the distorted prosthesis was accompanied by incus and/or malleus pathology. Of the remaining 9 of the 28 cases with previous ossiculoplasty, 5 had a correctly placed piston but pathology on the remaining ossicles, while 4 had no previous piston placement but had missing or fixed ossicles due to previous cholesteatoma surgery (2 cases), trauma (1) or tympanosclerosis (1).

\section{Otosclerosis cases}

Of the 21 otosclerosis patients who had undergone previous stapedoplasty, 11 (52 per cent) had incus erosion (most commonly at the level of the crimping of the previous prosthesis), while 3 had incus fixation within the epitympanum and 1 had congenital incus malformation. The malleus was partially or totally fixed at the level of the anterior malleal ligament in 5 patients (24 per cent), whereas the incudostapedial joint was found to be subluxated in 3 patients. Few patients had multiple reasons for failure. The previously placed pistons comprised 10 platinum-Teflon stapes prostheses, 4 titanium pistons and 4 undetermined prostheses; 3 cases did not have pistons.

\section{Audiometry results}

Pure tone averages (PTAs) for 0.5, 1, 2 and $3 \mathrm{kHz}$ were calculated for all 60 patients, over a mean follow-up period of 2.5 years. This demonstrated a mean preoperative air-bone gap (ABG) of $27.5 \mathrm{~dB}$ and a mean post-operative $\mathrm{ABG}$ of $11.3 \mathrm{~dB}$. Overall, 90 per cent of patients remained within $20 \mathrm{~dB}$ and 48 per cent within $10 \mathrm{~dB}$.

Primary malleostapedotomy patients performed slightly better than revision surgery patients, with a mean post-operative $\mathrm{ABG}$ of $9.4 \mathrm{~dB} ; 100$ per cent were 


\begin{tabular}{|c|c|c|c|c|c|c|c|}
\hline & PRE- AI & ST-MALLE & $\begin{array}{l}\text { III } \\
\text { EDOTON }\end{array}$ & $3 \mathrm{G}$ RES & & & \\
\hline Parameter & $0.5-3 \mathrm{kHz}$ & $0.5-4 \mathrm{kHz}$ & $0.5 \mathrm{kHz}$ & $1 \mathrm{kHz}$ & $2 \mathrm{kHz}$ & $3 \mathrm{kHz}$ & $4 \mathrm{kHz}$ \\
\hline Primary surgery & & & & & & & \\
\hline Pre-op ABG (mean; dB) & 26.3 & 28.8 & 40 & 30 & 15 & 15 & 25 \\
\hline Post-op ABG (mean; dB) & 9.4 & 10.6 & 10 & 12.5 & 5 & 5 & 15 \\
\hline Pts with $\mathrm{ABG}$ closure & & & & & & & \\
\hline$-<10 \mathrm{~dB}(\%)$ & 61 & 50 & 64 & 50 & 86 & 43 & 43 \\
\hline$-<20 \mathrm{~dB}(\%)$ & 100 & 93 & 93 & 93 & 100 & 68 & 71 \\
\hline Revision surgery & & & & & & & \\
\hline Pre-op ABG (mean; dB) & 27.5 & 30 & 30 & 30 & 20 & 20 & 32.5 \\
\hline Post-op ABG (mean; dB) & 11.3 & 13.8 & 15 & 15 & 5 & 10 & 20 \\
\hline Pts with ABG closure & & & & & & & \\
\hline$-<10 \mathrm{~dB}(\%)$ & 38 & 34 & 47 & 47 & 75 & 63 & 34 \\
\hline$-<20 \mathrm{~dB}(\%)$ & 81 & 78 & 66 & 84 & 88 & 88 & 69 \\
\hline
\end{tabular}

within $20 \mathrm{~dB}$ and 61 per cent were within $10 \mathrm{~dB}$ (Table III, Figure 2). In patients undergoing revision surgery, the mean post-operative $\mathrm{ABG}$ was $11.3 \mathrm{~dB} ; 81$ per cent were within $20 \mathrm{~dB}$ and 38 per cent within $10 \mathrm{~dB}$ (Figure 3). Only one patient had an ABG worse than $30 \mathrm{~dB}$; this patient had an underlying myringosclerosis following tympanoplasty for a central perforation.

There was no statistically significant difference between the audiological outcomes of the primary and revision cases (numbers too small; $p=0.08$ for $0.5-3 \mathrm{kHz}$ ). Multiple statistical analyses revealed no significant difference in functional outcome between patients with differing aetiologies or ossicular pathologies. In addition, there was no significant difference between the hearing results of otosclerosis patients and patients with other pathologies. Hearing results were stable over time.

The post-operative bone conduction thresholds were within $5 \mathrm{~dB}$ of pre-operative values in 82 per cent of all malleostapedotomy patients, over the frequencies $0.5-3 \mathrm{kHz}$. In 15 per cent, an improvement (i.e. overclosure) was documented. Patients' bone conduction and $\mathrm{ABG}$ results at $4 \mathrm{kHz}$ were found to be worse than at any other frequency. In one-third of all patients, bone conduction worsened by more than $5 \mathrm{~dB}$ at $4 \mathrm{kHz}$. Significant inner ear damage was observed in two patients ( 3 per cent). In one patient, manual perforation of a mobile footplate resulted in a loss of $25 \mathrm{~dB}$ bone conduction at 0.5 and $1 \mathrm{kHz}$. In another patient, a thin footplate fractured during perforation, requiring mobilisation of a small fragment with a $0.2 \mathrm{~mm}$ footplate elevator; this patient's bone conduction dropped by $20 \mathrm{~dB}$. No patient suffered complete loss of sensorineural hearing, and no patient in the primary malleostapedotomy group showed deterioration of bone conduction post-operatively.

No patient complained of vertigo or taste loss, judging from the most recent follow-up results available. The presence or absence of immediate post-operative imbalance was not recorded and therefore could not be evaluated. However, the most senior author's (TL) experience was that approximately $15-20$ per cent of patients did have some imbalance or dizziness during the first 3 days

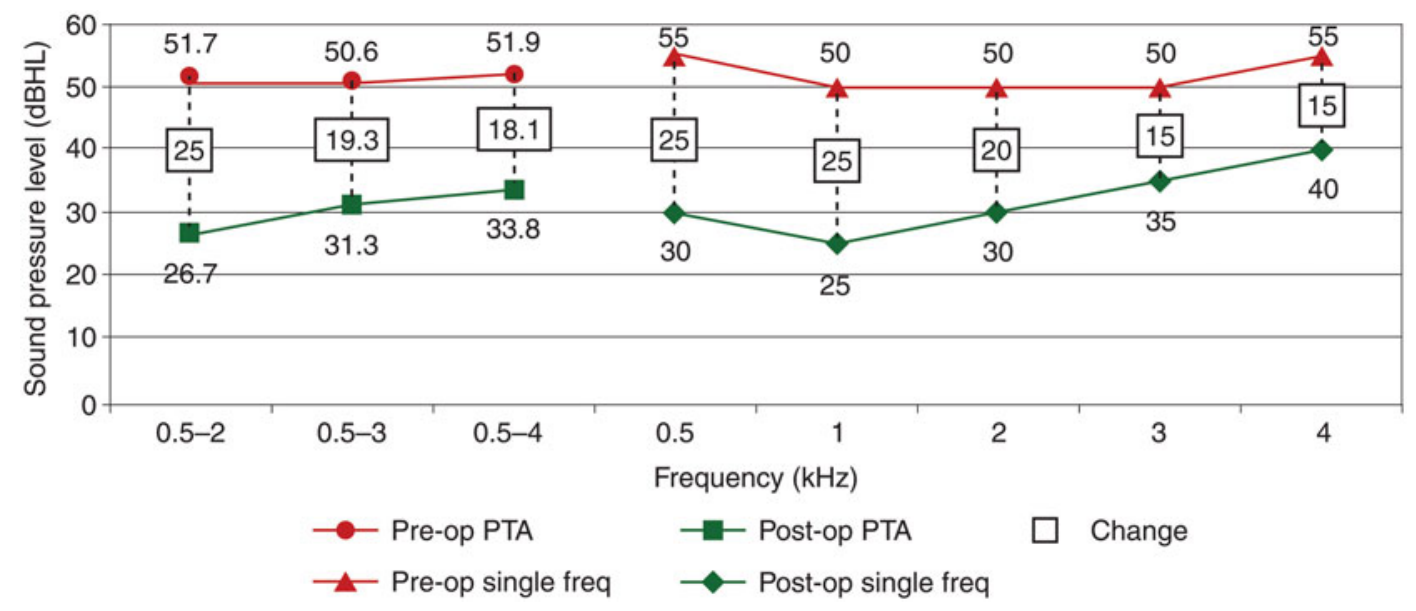

FIG. 2

Line graph showing changes in air conduction thresholds following primary malleostapedotomy. Pre-op $=$ pre-operative; PTA $=$ pure tone average; freq $=$ frequency; Post-op $=$ post-operative. 


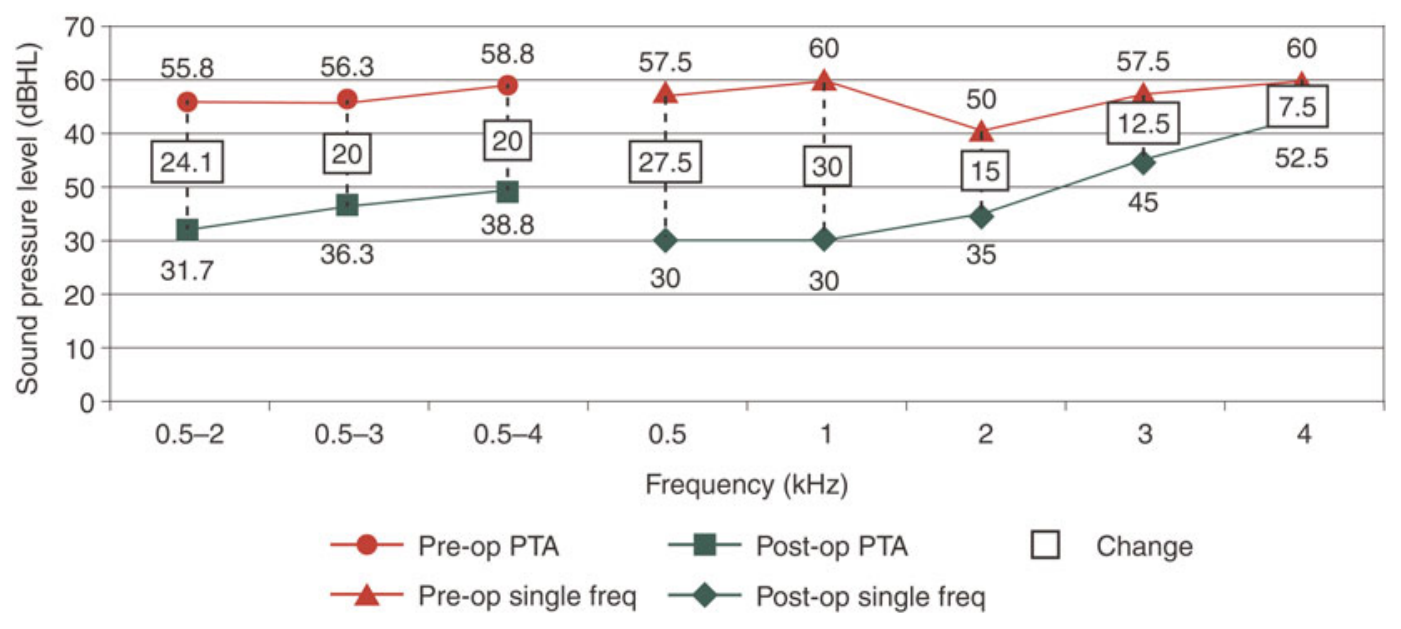

FIG. 3

Line graph showing changes in air conduction thresholds following revision malleostapedotomy. Pre-op $=$ pre-operative; PTA = pure tone average; freq $=$ frequency; Post-op $=$ post-operative .

after malleostapedotomy (usually on days 1-3 rather than on the day of surgery), without requiring any specific treatment.

There was no statistically significant difference between the ABGs calculated from the first postoperative PTA (performed more than three weeks but less than six months after surgery) versus the most recent post-operative PTA. There was also no difference in the ABG or bone conduction recorded for prostheses of different lengths.

\section{Discussion}

Various techniques have been described to connect the malleus to the stapes and the vestibule. Whereas malleostapediopexy connects the malleus to an intact and mobile stapes head, ${ }^{8}$ malleus-to-footplate prosthetic interpositions connect the malleus to an intact and mobile footplate even in the presence of a stapes suprastructure. ${ }^{9}$ The stapedectomy procedure which creates a direct connection between the malleus and the vestibule through the oval window has been termed an incus bypass procedure by Sheehy, malleovestibulopexy by Plester and malleus grip prosthesis by Schuknecht. ${ }^{1,2}$ The transcanal technique using the middle third of the malleus handle was modified by Fisch, who used an endaural approach and the attachment of his titanium stapes piston just distal to the lateral process of the malleus; he termed this procedure a malleostapedotomy. ${ }^{3,6}$ The initial malleus grip prostheses of Schuknecht (fat-wire and later Teflon-wire pistons) were further customised using platinumTeflon prostheses (most recently modified to incorporate a Fisch titanium piston), a Kurz titanium malleovestibulopexy clip piston (with and without a ball joint) and a Gyrus Nitinol Teflon piston. Kwok and Fisch ${ }^{7}$ examined the morphology and diameter of the malleus handle in order to determine the ideal placement of the piston so as to avoid dislocation into or out of the vestibule upon maximum excursion of the tympanic membrane; positioning just distal to the short process of the malleus handle was thought best. This required minimal elevation of the tympanic membrane and an anterior extension of the tympanomeatal flap design. ${ }^{6}$ Comparison of different pistons indicated that the Storz Fisch titanium stapes piston allowed a firm connection to the malleus handle close to the lateral process without slippering along the decreasing diameter of the malleus toward the umbo. ${ }^{7}$ In cases with a slim and oval-shaped malleus, we have further modified the ideal placement of the clip portion by drilling a small, flat surface onto the malleus handle using a $0.6 \mathrm{~mm}$ drill. Additional benefits of the titanium prosthesis comprise the facility for individual length adjustment using a universal cutting block, and the facility to bend the piston shaft to allow perpendicular placement of the prosthesis into the stapedotomy opening. These were the reasons why this technique and this prosthesis were used in all patients in the current series.

Historically, malleostapedotomy was mainly used for revision surgery, initially to correct unsuccessful fenestration procedures and later for revision stapes procedures. Most studies analysing intra-operative findings during revision stapes surgery for otosclerosis have identified prosthesis displacement and incus erosion as the most frequent events. ${ }^{4,10}$ The impact of hyalinisation of the anterior malleal ligament on malleus mobility was investigated by Nandapalan et al., ${ }^{5}$ and led to the development of the malleostapedotomy concept advocated by Fisch.

In our series, we found an even higher incidence of incus fixation within the epitympanum or possibly at the level of the posterior process. Partial fixation of the incus and malleus may be overlooked at primary surgery if the exposure of the ossicles is too limited. The endaural approach with a supero-anterior canalplasty permits the inspection of the anterior malleal process and ligament in all cases. With the systematic 
use of this exposure in the present series of primary malleostapedotomy procedures, the malleus was found to be fixed in 36 per cent of cases. The high rate of impaired incus mobility ( 70 per cent overall) was rather unexpected and requires further confirmation. This incidence may be slightly overestimated, since we did not open the epitympanum to identify the fixation point, and an additional malleus fixation may have been broken or mobilised upon careful luxation of the incus before re-testing the mobility of the malleus. In our revision cases for pure otosclerosis, the malleus mobility was impaired in 24 per cent and the incus mobility in 14 per cent; in addition, subluxation within the incudomalleal joint was seen in 14 per cent. Huber et al. ${ }^{11}$ found that partial fixation of the anterior malleal ligament, in the temporal bone laboratory, led to a persistent $\mathrm{ABG}$ of $10 \mathrm{~dB}$, and may account for some failures after primary stapes surgery. ${ }^{11}$ In a finite element ear model, Dai et al. ${ }^{12}$ calculated that the impact of fixation of both the superior and anterior malleal ligaments was $15 \mathrm{~dB}$ hearing loss in the low frequencies.

Fisch et al. ${ }^{4}$ analysed 80 cases of revision surgery for otosclerosis, and found partial and total malleus fixation in 37.5 and 8.7 per cent, respectively, and incus fixation in 13.7 per cent. Schmid and Häusler ${ }^{13}$ assessed clinical findings in 201 revision stapes surgery cases in which the anterior malleal process and ligament were not systematically inspected, and found incus or malleus head fixation in 8 per cent. Vincent et al. ${ }^{10}$ studied revision stapes surgery cases and found malleus ankylosis in only 3.4 per cent of 652 patients. Even though patient selection differed in these studies, there still seems to be a substantial discrepancy between the incidence of incus and malleus head fixation. Nandapalan et $\mathrm{al} .{ }^{5}$ examined otosclerotic temporal bones and identified a significantly greater incidence of hyalinisation of the anterior malleal ligament, compared with normal, non-otosclerotic temporal bones. These fixations did not correlate with the extent of the otosclerotic focus but were age-dependent, indicating that a longer duration of otosclerotic stapes fixation may lead to increased malleal ligament hyalinisation.

In order to contextualise our patients' audiological outcomes, we identified 20 published reports on the results of incus bypass procedures, malleovestibulopexy and malleostapedotomy, which summarised findings in groups ranging from 5 to 64 patients (apart from the initial reports by Schuknecht and Bartley and Sheehy, which included over 180 patients). The more recent results were comparable to our own, with closure of the ABG to within $20 \mathrm{~dB}$ in $70-90$ per cent. Closure to within $10 \mathrm{~dB}$ showed some variability, being achieved in between 20 and 70 per cent of cases. The best results were observed at the lower frequencies of $0.5-1 \mathrm{kHz}$, while worse $\mathrm{ABGs}$ and bone conduction results were found at $4 \mathrm{kHz}$. Fisch et al. $^{4}$ found a significantly better functional result for malleostapedotomy, compared with standard incus-stapedotomy, at 0.5 and $1 \mathrm{kHz}{ }^{4}$

- Use of a modified malleostapedotomy in primary and revision surgery was assessed

- An enlarged skin flap incision and a partial anterosuperior canalplasty allowed better malleus exposure and access

- This technique should be used for malleus or incus pathology combined with footplate fixation

- Results are equivalent to or better than standard incus-stapedotomy

Our favourable results for primary malleostapedotomy encourage the use of this technique in any case of even partial fixation of the incus and/or malleus in the presence of stapes footplate fixation. The same is true in revision cases. Most frequently, an incus erosion or a dislocation of the previous piston is observed at surgery. Removing the incus enlarges markedly the surgeon's view of the previously perforated and scarred footplate. In cases with normal malleus mobility, the head does not need further dissection, while in cases with partial fixation, the head can easily be sectioned using a $0.8 \mathrm{~mm}$ diamond drill or a malleus nipper. ${ }^{14}$

In our study, 81 per cent of patients achieved $A B G$ closure to within $20 \mathrm{~dB}$, and we observed no significant difference between primary and revision malleostapedotomy cases. Our results do not contradict those of Fisch et al., ${ }^{4}$ who found that patients undergoing their first revision procedure had a better chance of ABG closure to $0-20 \mathrm{~dB}$ (achieved in 84 per cent) than those undergoing their second or third revision procedure. Using this classification, most of our patients would have been in the first revision procedure group.

\section{Conclusion}

In this study, malleostapedotomy was performed using the malleable and adjustable Storz Fisch titanium piston and exposing the proximal handle of the malleus via an endaural approach. This permitted proper fixation of the piston in both primary and revision cases, and provided a wide view over the fixed and possibly scarred footplate, allowing perpendicular positioning of the prosthesis shaft into the vestibule. These modifications in surgical exposure and prosthesis design enabled us to reduce the unpleasant consequences of the initial malleus grip prosthesis, from a reported incidence of profound sensorineural hearing loss of 8 per cent to less than 3 per cent, and to obtain results equivalent or even superior to those of standard incus-stapedotomy.

In patients with partial hyalinisation of the ligaments of both the incus and the malleus, the proper evaluation 
of ossicular chain mobility remains a diagnostic challenge. We encourage further research into the evaluation of incus and malleus mobility in patients undergoing ossiculoplasty.

\section{References}

1 Schuknecht HF, Bartley ML. Malleus grip prosthesis. Ann Otol Rhinol Laryngol 1986;95:531-4

2 Sheehy JL. Stapedectomy: incus bypass procedures. A report of 203 operations. Laryngoscope 1982;92:258-62

3 Fisch U, May JS. Tympanoplasty, Mastoidectomy, and Stapes Surgery. Stuttgart: Georg Thieme Verlag, 1994

4 Fisch U, Acar FÖ, Huber A. Malleostapedotomy in revision surgery for otosclerosis. Otol Neurotol 2001;22:776-85

5 Nandapalan V, Pollak A, Langner A, Fisch U. The anterior and superior malleal ligaments in otosclerosis: a histopathologic observation. Otol Neurotol 2002;23:854-61

6 Fisch U, May JS, Linder T. Tympanoplasty, Mastoidectomy and Stapes Surgery, 2nd edn. Stuttgart: Georg Thieme Verlag, 2008

7 Kwok P, Fisch U, Nussbaumer M, Herkenhoff S, Strutz J. Morphology of the malleus handle and the comparison of different prostheses for malleostapedotomy. Otol Neurotol 2009;30: $1175-85$

8 Yuge K, Daito K, Nakamura K, Murakami M, Hattori Y, Murakami T et al. Results of malleostapediopexy. Auris Nasus Larynx 1982;9:145-50

9 Colletti V, Fiorino FG. Malleus-to-footplate prosthetic interposition: experience with 265 patients. Otolaryngol Head Neck Surg 1999;120:437-44
10 Vincent R, Rovers M, Zingade N, Oates J, Sperling N, Deveze A et al. Revision stapedotomy: operative findings and hearing results. A prospective study of 652 cases from the otology-neurotology database. Otol Neurotol 2010;31: $875-82$

11 Huber A, Koike T, Wada H, Nandapalan V, Fisch U. Fixation of the anterior mallear ligament: diagnosis and consequences for hearing results in stapes surgery. Ann Otol Rhinol Laryngol 2003;112:348-55

12 Dai C, Cheng T, Wood MW, Gan RZ. Fixation and detachment of superior and anterior malleolar ligaments in human middle ear: experiment and modeling. Hear Res 2007;230:24-33

13 Schmid P, Häusler R. Revision stapedectomy: an analysis of 201 operations. Otol Neurotol 2009;30:1092-100

14 Fisch U, Linder T. Temporal Bone Dissection, the Zurich Guidelines. Tuttlingen: Endo-Press, 2005

Address for correspondence:

Prof Thomas Linder,

Department of Otorhinolaryngology - Head and Neck Surgery,

Kantonsspital Luzern,

Spitalstrasse,

CH 6000 Lucerne, Switzerland

Fax: +41412054995

E-mail: Thomas.Linder@luks.ch

Professor T Linder takes responsibility for the integrity of the content of the paper

Competing interests: None declared 